\title{
Early clinical and laboratory risk factors of intensive care unit requirement during 2004-2008 dengue epidemics in Singapore: a matched case-control study
}

\author{
Junxiong Pang ${ }^{1,2,3^{*}}$, Tun-Linn Thein ${ }^{3}$, Yee-Sin Leo ${ }^{1,3,4}$ and David C Lye $e^{3,4,5}$
}

\begin{abstract}
Background: Dengue infection can result in severe clinical manifestations requiring intensive care. Effective triage is critical for early clinical management to reduce morbidity and mortality. However, there is limited knowledge on early risk factors of intensive care unit (ICU) requirement. This study aims to identify early clinical and laboratory risk factors of ICU requirement at first presentation in hospital and 24 hours prior to ICU requirement.

Method: A retrospective 1:4 matched case-control study was performed with 27 dengue patients who required ICU, and 108 dengue patients who did not require ICU from year 2004-2008, matched by year of dengue presentation. Univariate and multivariate conditional logistic regression were performed. Optimal predictive models were generated with statistically significant risk factors identified using stepwise forward and backward elimination method.

Results: ICU dengue patients were significantly older ( $P=0.003)$ and had diabetes ( $P=0.031)$, compared with non-ICU dengue patients. There were seven deaths among ICU patients at median seven days post fever. At first presentation, the WHO 2009 classification of dengue severity was significantly associated $(P<0.001)$ with ICU, but not the WHO 1997 classification. Early clinical risk factors at presentation associated with ICU requirement were hematocrit change $\geq 20 \%$ concurrent with platelet $<50 \mathrm{~K}$ [95\% confidence-interval $(\mathrm{Cl})=2.46-30.53$ ], hypoproteinemia ( $95 \% \mathrm{Cl}=1.09-19.74)$, hypotension ( $95 \% \mathrm{Cl}=1.83-31.79$ ) and severe organ involvement $(95 \% \mathrm{Cl}=3.30-331)$. Early laboratory risk factors at presentation were neutrophil proportion ( $95 \% \mathrm{Cl}=1.04-1.17)$, serum urea (95\% Cl=1.02-1.56) and alanine aminotransferase level (95\% $\mathrm{Cl}=1.001-1.06)$. This predictive model has sensitivity and specificity up to $88 \%$. Early laboratory risk factors at 24 hours prior to ICU were lymphocyte ( $95 \% \mathrm{Cl}=1.03-1.38)$ and monocyte proportions ( $95 \% \mathrm{Cl}=1.02-1.78)$, pulse rate $(95 \% \mathrm{Cl}=1.002-1.14)$ and blood pressure $(95 \% \mathrm{Cl}=0.92-0.996)$. This predictive model has sensitivity and specificity up to $88.9 \%$ and $78 \%$, respectively.

Conclusions: This is the first matched case-control study, to our best knowledge, that identified early clinical and laboratory risk factors of ICU requirement during hospitalization. These factors suggested differential pathophysiological background of dengue patients as early as first presentation prior to ICU requirement, which may reflect the pathogenesis of dengue severity. These risk models may facilitate clinicians in triage of patients, after validating in larger independent studies.
\end{abstract}

Keywords: Dengue, Intensive care unit, Early risk factors, Matched case-control

\footnotetext{
*Correspondence: Junxiong_Pang@nuhs.edu.sg

'Saw Swee Hock School of Public Health, National University of Singapore, Singapore, Singapore

${ }^{2}$ Infectious Diseases, Genome Institute of Singapore, Singapore, Singapore

Full list of author information is available at the end of the article
}

\section{Biomed Central}

(c) 2014 Pang et al.; licensee BioMed Central Ltd. This is an Open Access article distributed under the terms of the Creative Commons Attribution License (http://creativecommons.org/licenses/by/2.0), which permits unrestricted use, distribution, and reproduction in any medium, provided the original work is properly credited. The Creative Commons Public Domain Dedication waiver (http://creativecommons.org/publicdomain/zero/1.0/) applies to the data made available in this article, unless otherwise stated. 


\section{Background}

Dengue is identified as the most rapidly spreading mosquito-borne viral disease in the world by the World Health Organization (WHO) in 2012 [1]. Approximately $70 \%$ of the population at risk is residing in the WHO Southeast Asia and Western Pacific regions, which make up about $75 \%$ of the current global dengue disease burden. This has led to the formation of the Asia Pacific Dengue Strategic Plan (2008-2015) and the recent Global Strategy for Prevention and Control (2012-2020) to reduce the dengue burden [1].

Dengue results in a wide spectrum of non-specific clinical manifestations with unpredictable clinical course and outcome. Patients can be clinically classified as 'dengue fever' (DF), 'dengue hemorraghic fever' (DHF) or 'dengue shock syndrome' (DSS) according to the 1997 WHO dengue classification system and it can be largely asymptomatic [2]. Recently, patients can be clinically classified as 'probable dengue', 'dengue with warning signs' or 'severe dengue' according to the 2009 WHO classification system [3]. Although a large proportion of patients recover after a mild self-limiting disease, a small proportion may progress to develop severe dengue clinical manifestations, which require interventions in intensive care unit (ICU). The progression to severe clinical manifestations is usually unpredictable [3]. Without prompt and appropriate therapy, case fatality rate may exceed $20 \%$ [3].

There are currently no systematic studies, but only case series reported [4-6] focusing on epidemiological, clinical and laboratory risk factors at first presentation in hospital that are predictive of clinical severity, as defined by the requirement for ICU, instead of the WHO classification criteria. Generally, the proportion of hospitalized dengue patients requiring ICU is low (0.1\%-9\%) among the high number of hospitalized cases reported [4-7], including in Singapore $[8,9]$. This is likely due to the fact that there is less severe manifestations in adult dengue patients as compared to pediatric dengue patients, whom are at higher risk of ICU [10]. Furthermore, the timely diagnosis and supportive dengue care management in a more established healthcare system, such as in Singapore may have also resulted in only a small proportion of dengue cases who require ICU admission. Therefore, case series studies and classification may not be generalizable to all populations at risk as well as in different clinical setting and healthcare system. Moreover, with the recommendation of close monitoring of patients with warning signs in hospital [3], there is a greater need to identify these high risk dengue patients 24 hours prior to ICU requirement during hospitalization, which is currently lacking. Henceforth, this study aims to identify and evaluate the early clinical and laboratory risk factors of ICU requirement at first presentation in hospital as well as 24 hours prior to ICU requirement.

\section{Methods}

A retrospective matched case-control study was conducted using anonymized data collected from all adult dengue patients admitted into intensive care unit (ICU) from 1 January 2004 to 31 December 2008 to the Department of Infectious Diseases at Tan Tock Seng Hospital (TTSH). This is the largest hospital in Singapore for the treatment of dengue patients where dengue patients were managed using a standardized dengue care path. Hospitalization criteria were defined in previous published study [8]. In Singapore, dengue infections were predominantly due to dengue serotype 1 (detected in $75 \%$ to $100 \%$ of dengue samples collected each month) during the epidemics in the year 2004 to 2006, and dengue serotype 2 (detected in up to $91 \%$ of dengue samples) during the epidemic in the year 2007 and 2008 [11]. Each case who were admitted into ICU was randomly matched to four patients who did not require intensive care by the year of dengue presentation as controls. Dengue-confirmed patients with positive dengue polymerase chain reaction (PCR) assay, and probable dengue patients with positive dengue immunoglobulin-M (IgM) or immunoglobulin-G (IgG) (Dengue Duo IgM \& IgG Rapid Strip, Panbio Diagnostic, Queensland, Australia) and fulfilling either the WHO 1997 or 2009 probable dengue criteria, were included in this study.

According to the WHO 1997 criteria [2], dengue fever (DF) was defined as fever with any two of the following manifestations: headache, retro-orbital pain, myalgia, arthralgia, rash, hemorrhagic manifestations, or leucopenia. Dengue hemorrhagic fever (DHF) was diagnosed when all four criteria of fever, hemorrhagic manifestations, thrombocytopenia $\left(<100 \times 10^{9} / \mathrm{L}\right)$ and evidence of plasma leakage (hematocrit change $\geq 20 \%$, hypoproteinemia or clinical fluid accumulation) were present. Dengue shock syndrome was diagnosed with the presence of rapid and weak pulse with narrow pulse pressure $<20 \mathrm{mmHg}$, or hypotension in a patient with DHF. Probable dengue by WHO 2009 criteria [3] was defined as fever and two of the following: nausea or vomiting, rash, aches and pain, leucopenia, and any warning sign. Warning signs were abdominal pain or tenderness, persistent vomiting, clinical fluid accumulation, mucosal bleed, lethargy or restlessness, hepatomegaly, and hematocrit change $\geq 20 \%$ concurrent with platelet $<50 \mathrm{~K}$ on the same day. The WHO warning sign of rapid change in hematocrit with rapid drop in platelet count was qualitative. Hence, we had explored previously $[9,12]$ using a different quantitative definition of hematocrit change of $\geq 20 \%$ with concurrent platelet nadir $<50 \mathrm{~K}$, which is more practical to implement in clinical setting, and had been associated with death outcome [12]. Severe dengue cases fulfil at least one of three criteria: (1) severe plasma leakage: associated with shock (tachycardia $>100$ or narrow pulse pressure $<20$ or blood 
pressure $<90 \mathrm{mmHg}$ ), (2) severe bleeding: gastrointestinal tract bleeding such as hematemesis or melena or bleeding per rectum, pack cell transfusion or blood transfusion requirement or menorrhagia, (3) severe organ impairment: defined as aspartate or alanine transaminase $\geq 1000$ units/l, encephalopathy, myocarditis and acute renal impairment (creatinine $\times 2$ upper limit of normal for age and sex; based on modification of diet in renal disease equation for glomerular filtration rate $=75 \mathrm{ml} / \mathrm{min}$ or baseline estimated from minimum creatinine recorded).

The data at first presentation in hospital and 24 hours prior to ICU requirement were obtained from medical records: demographic, epidemiological, co-morbidity, clinical, laboratory, treatment and outcome data. The severity of the patients as evaluated by the WHO 1997 and 2009 classifications was determined at first presentation and during hospitalisation. The duration of progression to 'DHF' or 'severe dengue' was determined only for patients who were either classified as 'DF' or 'probable dengue with/without warning signs' at first presentation. The number of days post presentation (DPP) was used to define the period since the first presentation in hospital. The number of days post fever (DPF) was used to define the period since the day of fever onset.

\section{Statistical methods}

Univariate and multivariate conditional logistic regression were performed to assess the association between the variables and dengue severity as defined by ICU requirement. Conditional logistic regression was used to account for the matching factor selected in the analyses [13]. Matching was performed based on the year of presentation to control for potential confounding by the predominant serotype in each year. Confounding effect was further minimized by performing multivariate conditional logistic regression adjusting for significant demographic \& co-morbidity factors associated with ICU requirement only. This parsimonious approach would minimize overloading the multivariable model to achieve higher statistical power to detect true association as the sample size available is small $[14,15]$. Continuous variables were illustrated in median with the interquartile range (IQR) for reference. The laboratory variables were analyzed in the continuous format to maximize all the data available, and to minimize reporting bias, especially when the variables were categorized into the expected normal or hypothetical range. For analysis of early risk factors 24 hour prior to ICU requirement, four ICU patients were excluded as they were admitted into ICU less than 24 hour post presentation to hospital. The most optimal predictive model was derived using the stepwise forward and backward elimination method, with the top 10 most significantly associated variables based on the $P$-value of the univariate conditional regression analyses. Only variables with
$P$-value $<0.05$ would remain in the optimal model during the model development. Hosmer-Lemeshow Goodness of fit test was used to check if the model fit the observed dataset with $P>0.05$. Likelihood ratio test was used to assess if a model is more efficient than the other similar model with more significant variables. Fisher's exact test and Wilcoxon rank-sum test were used for categorical and continuous variables, respectively, for the subgroup analysis of death cases in ICU group. All statistical analyses were performed using Stata 10.0 (Stata Corp., College Station, TX, 2005). All tests were conducted at the 5\% level of significance, with conditional odds ratio (COR) and/or adjusted COR (ACOR), $P$-value and corresponding 95\% CI reported where applicable.

\section{Ethics statement}

This study was approved by Domain Specific Review Board, National Healthcare Group, Singapore (DSRB-E/ 08/567) with waiver of informed consent as data were analyzed anonymously.

\section{Results}

A total of 8,123 dengue-infected patients were admitted into Tan Tock Seng Hospital during the dengue epidemics between January 2004 and December 2008, with national incidence rate (per 100,000 population) of about 210 in 2004, 320 in 2005, 70 in 2006, 190 in 2007, and 160 in 2008 [16]. Among these admissions, there were a total of 27 dengue-infected cases $(0.3 \%)$ that required intensive care interventions in the intensive care unit (ICU). A total of 108 dengue-infected matched controls that did not require intensive care interventions were involved. The median age of the cases was 44 years [Interquartile range (IQR): 36-53 years], with 30\% female and $78 \%$ Chinese. The median age of the controls was 34 years (IQR: $25-44$ years), with $36 \%$ female and $71 \%$ Chinese (Table 1). Among the cases, there were $48 \%$ positive for dengue polymerase chain reaction assay (PCR) and 52\% were serology positive. Among the controls, there were $29 \%$ positive for dengue PCR, and $71 \%$ were serology positive. The median days post fever (DPF) at presentation was 3 (IQR: $3-5$ days) and 5 days (IQR: 4-5 days) for cases and controls, respectively. Patients requiring ICU were sicker, requiring earlier admission to hospital. However, there was no statistically significant association observed in gender, ethnic groups, IgG status and median DPF at first presentation with ICU requirements.

Out of these 27 ICU cases, there were about 93\% $(n=25)$ who had severe plasma leakage, with $81 \%(n=22)$ having shock, 37\% $(\mathrm{n}=10)$ had severe bleeding, and 56\% $(\mathrm{n}=16)$ had severe organ impairment involving renal, liver and central nervous system during hospitalization. Among the seven death cases, 100\% had severe plasma leakage, 71\% 
Table 1 Demographic and co-morbidities of dengue-infected non-ICU (controls) and ICU (cases) patients

\begin{tabular}{|c|c|c|c|c|c|c|c|c|c|c|}
\hline Variables & $\begin{array}{l}\text { Den-infected } \\
\text { non-ICU controls } \\
(n=108)\end{array}$ & $\% /(I Q R)$ & $\begin{array}{l}\text { Den-infected } \\
\text { ICU cases } \\
(\mathrm{n}=27)\end{array}$ & $\% /($ IQR) & COR & P-value & $95 \% \mathrm{Cl}$ & ACOR $^{*}$ & P-value & $95 \% \mathrm{Cl}$ \\
\hline Median age & 34 & $(25-44)$ & 44 & $(36-53)$ & 1.07 & 0.003 & $1.02-1.11$ & 1.05 & 0.014 & $1.01-1.10$ \\
\hline \multicolumn{11}{|l|}{ Age groups } \\
\hline $14-30$ & 36 & 33 & 4 & 15 & 1 & & & & & \\
\hline $30-39$ & 32 & 30 & 7 & 26 & 3.64 & 0.138 & $0.66-20.0$ & 2.48 & 0.244 & $0.54-11.41$ \\
\hline $40-49$ & 24 & 22 & 7 & 26 & 5.9 & 0.061 & $0.92-37.9$ & 4.1 & 0.126 & $0.67-25.04$ \\
\hline $50-59$ & 13 & 12 & 6 & 22 & 8.51 & 0.021 & $1.38-52.6$ & 6.9 & 0.027 & $1.25-38.23$ \\
\hline$\geq 60$ & 3 & 2.8 & 3 & 11 & 21.98 & 0.012 & $1.97-245.2$ & 5.49 & 0.165 & $0.50-60.54$ \\
\hline \multicolumn{11}{|l|}{ Gender } \\
\hline Female & 39 & 36 & 8 & 30 & 0.74 & 0.52 & $0.29-1.87$ & 0.59 & 0.318 & $0.21-1.65$ \\
\hline \multicolumn{11}{|l|}{ Ethnic groups } \\
\hline Chinese & 77 & 71 & 21 & 78 & 1 & & & & & \\
\hline Malay & 5 & 4.6 & 2 & 7.4 & 1.44 & 0.667 & $0.28-7.49$ & 2.47 & 0.36 & $0.36-17.15$ \\
\hline Indian & 15 & 14 & 2 & 7.4 & 0.45 & 0.344 & $0.09-2.37$ & 0.39 & 0.315 & $0.06-2.47$ \\
\hline Others & 11 & 10 & 2 & 7.4 & 0.65 & 0.606 & $0.13-3.36$ & 0.95 & 0.952 & $0.17-5.29$ \\
\hline \multicolumn{11}{|l|}{ lgG at presentation } \\
\hline $\operatorname{lgG}+$ & 42 & 39 & 12 & 44 & 1.26 & 0.596 & $0.53-2.98$ & 1.19 & 0.708 & $0.47-3.03$ \\
\hline Median DPF at presentation & 5 & $(4-5)$ & 3 & $(3-5)$ & 0.75 & 0.059 & $0.56-1.01$ & 0.78 & 0.112 & $0.58-1.06$ \\
\hline \multicolumn{11}{|l|}{ Diabetes mellitus } \\
\hline Yes & 3 & 2.8 & 4 & 15 & 11.57 & 0.031 & $1.25-1077$ & 5.53 & 0.142 & $0.56-54.15$ \\
\hline \multicolumn{11}{|l|}{ Hypertension } \\
\hline Yes & 10 & 9.3 & 5 & 19 & 2.22 & 0.184 & $0.69-7.17$ & 0.42 & 0.296 & $0.08-2.14$ \\
\hline \multicolumn{11}{|l|}{ Hyperlipidemia } \\
\hline Yes & 7 & 6.5 & 4 & 15 & 2.57 & 0.168 & $0.67-9.83$ & 1 & 0.998 & $0.20-4.87$ \\
\hline \multicolumn{11}{|l|}{ Cardiac disorder } \\
\hline Yes & 2 & 1.9 & 3 & 11 & 9.83 & 0.051 & $0.99-97.23$ & 3.18 & 0.402 & $0.21-47.52$ \\
\hline
\end{tabular}

DPF- Days Post Fever; COR- Conditional Odds Ratio; IQR- Interquartile range.

*Adjusted Conditional Odds Ratio (ACOR) was obtained from a multivariate conditional logistic regression with test variables being adjusted by age and diabetes mellitus.

Data in bold are risk factors significantly associated with ICU requirement.

had severe bleeding, and 100\% had severe organ involvement. More descriptions can be found in Additional file 1: Table S1 and in a previously published dengue death study [12]. All ICU patients had intravenous (IV) fluid of about 15 liters on average during hospitalization, and about $40 \%$ of the ICU patients had also IV fluid before progression to DHF/DSS. On the contrary, $86 \%$ of nonICU patients had IV fluid of about 4.6 liters on average during hospitalization, and only about $8.6 \%$ had IV fluid before progression to DHF/DSS. About $63 \%$ of the ICU patients had received platelets infusions, while only $9.7 \%$ of the non-ICU patients had received platelets infusions. About 22\% of the ICU patients had blood transfusion and only about $1 \%$ of the non-ICU patients had blood transfusion. About $30 \%$ of the ICU patients had required mechanical ventilation, and none required for the non-ICU patients.
Demographic and co-morbidity risk factors of ICU

Age groups 50-59 years old [conditional odds ratio $(\mathrm{COR})=8.51 ; 95 \%$ confidence interval $(\mathrm{CI})=1.38-52.6$ ] and equal to or greater than 60 years old $(\mathrm{COR}=21.98$; $95 \% \mathrm{CI}=1.97-245$ ) had significantly higher risk of ICU requirement compared with age group 14 to 30 years old. However, after adjusting for diabetes mellitus, only age group 50-59 years old was observed to be a statistically significant risk factor (adjusted COR $(\mathrm{ACOR})=6.9$; 95\% CI $=1.25-38.23$ ) of ICU requirement (Table 1 ). Among the co-morbidities analyzed, patients with diabetes mellitus was found to have significantly higher risk $(\mathrm{COR}=11.57 ; 95 \% \mathrm{CI}=1.25-1077)$ of $\mathrm{ICU}$ requirement compared with patients with no diabetes. After adjusting for age, the risk remained high with ACOR of 5.53 (95\% $\mathrm{CI}=0.56-54.15)$, but it was statistically not significant, probably due to the lack of statistical power with a small 
sample size (Table 1). In addition, patients with cardiac disorder were observed to be at higher risk $(\mathrm{ACOR}=$ 3.18; $95 \% \mathrm{CI}=0.21-47.52)$ compared with patients without. This may also be a potential risk factor that requires further investigation, even though it was statistically not significant in this study (Table 1).

\section{Dengue severity classification and clinical outcomes}

The WHO 1997 dengue severity classification at presentation was not significantly associated with ICU requirement $(\mathrm{ACOR}=1.8 ; 95 \% \mathrm{CI}=0.54-6.08)$, with sensitivity of about $19 \%$ and specificity of $87 \%$. However, the WHO 2009 dengue severity classification at presentation was significantly associated with ICU requirement $(\mathrm{ACOR}=$ 8.79; $95 \% \mathrm{CI}=2.65-29.16$ ) (Table 2), with sensitivity of $52 \%$ and specificity of $94 \%$. Both the WHO dengue severity classifications as final outcome were observed to be significantly associated with ICU requirement (WHO 1997: $\quad \mathrm{ACOR}=2.99 ; 95 \% \mathrm{CI}=1.15-7.78) \quad(\mathrm{WHO} 2009$ : ACOR $=116.4 ; 95 \% \mathrm{CI}=8.79-1541)($ Table 2). The WHO 1997 classification system as final outcome achieved sensitivity and specificity of $67 \%$ and $78 \%$, respectively. The WHO 2009 classification system achieved sensitivity and specificity of $89 \%$ and $90 \%$, respectively.

The median days of progression to DHF/DSS and severe dengue for cases was 4. (IQR: 3-5 days) and 3 Days
Post Presentation (DPP) (IQR: 3-5 days), respectively. For the controls, the median days was 3 (IQR: 2.33.8 days) and 2.5 DPP (IQR: 1.8-3.3 days). The median length of stay (LOS) in hospital was 8 (IQR: 6-13.5 days) and 4 days (IQR: $3-5$ days) for cases and controls, respectively. The median Days Post Fever (DPF) and DPP to ICU requirement was 6 (IQR: 4-7.5 days) and 3 (IQR: 2-4.5 days) days respectively. The median LOS in ICU for the cases was 3 days (IQR: 2-4 days). Among the cases, there were seven deaths (case fatality rate $=26 \%$ ), with median DPF of 7 days (IQR: 7-15.5 days) and median DPP of 3 days to death (IQR: 2.5-7.5 days) (Table 2).

\section{Early ICU risk factors at first presentation in hospital}

At first presentation, warning signs from the WHO 2009 classification were not observed to be significantly associated with ICU requirement. In addition, other signs and symptoms such as hemorrhagic manifestation, rash, leucopenia, nausea/vomiting, ache and pains, thrombocytopenia and tardycardia were not observed to be significantly associated with ICU requirement (Additional file 2: Table S2). Hypoproteinemia $(\mathrm{ACOR}=4.65 ; 95 \%$ $\mathrm{CI}=1.09-19.74)$, hypotension $(\mathrm{ACOR}=7.63 ; 95 \% \mathrm{CI}=$ 1.83-31.79), severe organ involvement $(\mathrm{ACOR}=33.04 ; 95 \%$ $\mathrm{CI}=3.30-331.3)$ and hematocrit change $\geq 20 \%$ concurrent

Table 2 Severity classification and duration of illness of dengue-infected non-ICU (controls) and ICU (cases) patients

\begin{tabular}{|c|c|c|c|c|c|c|c|c|c|c|}
\hline Variables & $\begin{array}{l}\text { Den-infected } \\
\text { non-ICU } \\
\text { controls } \\
(n=108) \\
\end{array}$ & $\% /(I Q R)$ & $\begin{array}{l}\text { Den-infected } \\
\text { ICU cases } \\
(\mathrm{n}=27)\end{array}$ & $\% /($ IQR) & COR & $\mathrm{P}$-value & $95 \% \mathrm{Cl}$ & ACOR $^{*}$ & P-value & $95 \% \mathrm{Cl}$ \\
\hline \multicolumn{11}{|l|}{ WHO 1997 (Presentation) } \\
\hline DHF/DSS & 14 & 13 & 5 & 19 & 1.5 & 0.469 & $0.50-4.4$ & 1.8 & 0.34 & $0.54-6.08$ \\
\hline \multicolumn{11}{|l|}{ WHO 2009 (Presentation) } \\
\hline Severe dengue & 7 & 6.5 & 14 & 52 & 10.3 & $<0.001$ & $3.70-28.84$ & 8.79 & $<0.001$ & $2.65-29.16$ \\
\hline \multicolumn{11}{|l|}{ WHO 1997 (Outcome) } \\
\hline DHF/DSS & 24 & 22 & 18 & 67 & 7.65 & $<0.001$ & $2.74-21.35$ & 2.99 & 0.025 & $1.15-7.78$ \\
\hline \multicolumn{11}{|l|}{ WHO 2009 (Outcome) } \\
\hline Severe dengue & 11 & 10 & 24 & 89 & 73.1 & $<0.001$ & $9.82-543.96$ & 116.4 & $<0.001$ & $8.79-1541$ \\
\hline Median DPP to DHF/DSS & 3 & $(2.3-3.8)$ & 4 & $(3-5)$ & 1.79 & 0.206 & $0.73-4.43$ & 3.08 & 0.677 & $0.02-608$ \\
\hline Median DPP to Severe dengue & 2.5 & $(1.8-3.3)$ & 3 & $(3-5)$ & & & & & & \\
\hline Median LOS in hospital & 4 & $(3-5)$ & 8 & $(6-13.5)$ & 1.73 & $<0.001$ & $1.30-2.32$ & 1.76 & 0.001 & $1.28-2.43$ \\
\hline Median DPF on ICU requirement & N.A & & 6 & $(4-7.5)$ & & & & & & \\
\hline Median DPP on ICU requirement & N.A & & 3 & $(2-4.5)$ & & & & & & \\
\hline Median LOS in ICU & N.A & & 3 & $(2-4)$ & & & & & & \\
\hline Death & 0 & 0 & 7 & 26 & & & & & & \\
\hline Median DPF to death & N.A & & 7 & $(7-15.5)$ & & & & & & \\
\hline Median DPP to Death & N.A & & 3 & $(2.5-7.5)$ & & & & & & \\
\hline
\end{tabular}

DPF- Days Post Fever; DPP- Days Post Presentation; LOS- Length of Stay; COR-Conditional Odds Ratio; IQR- Interquartile range.

*Adjusted Conditional Odds Ratio (ACOR) was obtained from a multivariate conditional logistic regression with test variables being adjusted by age and diabetes mellitus.

Data in bold are risk factors significantly associated with ICU requirement. 
with platelet $<50 \mathrm{~K}(\mathrm{ACOR}=8.66 ; 95 \% \mathrm{CI}=2.46-30.53)$ were observed to be significantly associated with ICU requirement (Table 3).

Pulse rate $(\mathrm{ACOR}=1.05 ; 95 \% \mathrm{CI}=1.01-1.09)$, neutrophil proportion $(\mathrm{ACOR}=1.11 ; 95 \% \mathrm{CI}=1.04-1.17)$, serum urea $(\mathrm{ACOR}=1.26 ; 95 \% \mathrm{CI}=1.02-1.56)$, creatinine $(\mathrm{ACOR}=$ 1.02; $95 \% \mathrm{CI}=1.01-1.04$ ), aspartate ( $\mathrm{ACOR}=1.01 ; 95 \%$ $\mathrm{CI}=1.001-1.06) \quad(\mathrm{AST})$ and alanine aminotransferases (ALT), $(\mathrm{ACOR}=1.01 ; 95 \% \mathrm{CI}=1.001-1.06)$ at first presentation were positively correlated with ICU requirement (Table 3). On the contrary, lymphocyte $(\mathrm{ACOR}=0.90$; $95 \% \mathrm{CI}=0.85-0.96)$ monocyte proportions $(\mathrm{ACOR}=0.86$; $95 \% \mathrm{CI}=0.77-0.95)$, platelet count $(\mathrm{ACOR}=0.98 ; 95 \%$ $\mathrm{CI}=0.96-0.99)$, serum protein $(\mathrm{ACOR}=0.88 ; 95 \% \mathrm{CI}=$ 0.79-0.98) and albumin (ACOR $=0.81 ; 95 \% \mathrm{CI}=0.70-0.94$ ) at first presentation were significantly and negatively correlated with ICU requirement (Table 3).

These early risk factors of ICU requirement at first presentation in hospital were used to create a predictive model.
The most optimal model derived was a combination of neutrophil proportion, ALT and serum urea level with an area under the curve (AUC) of 0.92 (Hosmer-Lemeshow Goodness of fit test $P=0.52$ ), indicating that the model had good discrimination of patients who are more likely to require ICU when screened at first presentation in hospital. A prognostic index $(P)$ was created using this model. Using the default cut-off of $P=0$, the model had sensitivity of $58.8 \%$, specificity of $96.3 \%$, positive predictive value (PPV) of $76.9 \%$ and negative predictive value (NPV) of 91.8\%. Using a cut-off of $P=-1.4$ (maximizing sensitivity and specificity), the model had sensitivity of $88.2 \%$, specificity of $88.9 \%$, PPV of $62.5 \%$ and NPV of $97.3 \%$. The predicted probability of ICU requirement $\left(p_{i}\right)$ at presentation was calculated as follows:

$$
\begin{aligned}
P_{\text {presentation }}=\ln \left(\frac{p i}{1-p i}\right)= & 0.106 x_{1 i}+0.004 x_{2 i}+0.326 x_{3 i} \\
& -10.601
\end{aligned}
$$

Table 3 Significantly associated clinical and laboratory variables at first presentation of dengue-infected ICU (cases)

\begin{tabular}{|c|c|c|c|c|c|c|c|c|c|c|}
\hline Variables & $\begin{array}{l}\text { Den-infected } \\
\text { non-ICU } \\
\text { controls } \\
(n=108)\end{array}$ & $\% /(I Q R)$ & $\begin{array}{l}\text { Den-infected } \\
\text { ICU cases } \\
(n=27)\end{array}$ & $\% /(I Q R)$ & COR & P-value & $95 \% \mathrm{Cl}$ & ACOR $^{*}$ & P-value & $95 \% \mathrm{Cl}$ \\
\hline \multicolumn{11}{|l|}{ Hypoproteinemia } \\
\hline Yes & 23 & 21 & 10 & 37 & 2.28 & 0.085 & $0.89-5.85$ & 4.65 & 0.037 & $1.09-19.74$ \\
\hline \multicolumn{11}{|l|}{ Hypotension } \\
\hline Yes & 4 & 3.7 & 7 & 26 & 8.62 & 0.002 & $2.21-33.63$ & 7.63 & 0.005 & $1.83-31.79$ \\
\hline \multicolumn{11}{|l|}{ Severe organ involvement } \\
\hline Yes & 1 & 0.9 & 10 & 37 & 40 & $<0.001$ & $5.12-312.5$ & 33.04 & 0.003 & $3.30-331.3$ \\
\hline \multicolumn{11}{|l|}{$\begin{array}{l}\text { Hematocrit change } \\
\geq 20 \% \text { concurrent } \\
\text { with platelet }<50 \mathrm{~K}\end{array}$} \\
\hline Yes & 7 & 6.5 & 11 & 41 & 9.4 & $<0.001$ & $2.95-29.83$ & 8.66 & 0.001 & $2.46-30.53$ \\
\hline Systolic blood pressure & 105 & $(100-114)$ & 110 & $(91.5-118.5)$ & 0.98 & 0.216 & $0.96-1.01$ & 0.97 & 0.046 & $0.94-0.99$ \\
\hline Pulse rate & 90 & $(80-96)$ & 95.5 & $(88.5-102)$ & 1.03 & 0.049 & $1.00-1.07$ & 1.05 & 0.012 & $1.01-1.09$ \\
\hline Proportion neutrophils & 60.3 & $(46.2-70.4)$ & 76.8 & $(70.7-81.5)$ & 1.11 & $<0.001$ & $1.05-1.18$ & 1.11 & 0.001 & $1.04-1.17$ \\
\hline Proportion lymphocytes & 30.3 & $(22-39)$ & 16.5 & $(9.8-19.9)$ & 0.90 & $<0.001$ & $0.85-0.95$ & 0.90 & 0.001 & $0.85-0.96$ \\
\hline Proportion monocytes & 12.5 & $(8.9-17.3)$ & 7.9 & $(5.1-9.9)$ & 0.84 & 0.002 & $0.76-0.94$ & 0.86 & 0.004 & $0.77-0.95$ \\
\hline Platelet & 68 & $(44-84)$ & 42.5 & $(20.3-74.8)$ & 0.98 & 0.01 & $0.96-0.99$ & 0.98 & 0.017 & $0.96-0.99$ \\
\hline Serum urea & 3.5 & $(2.6-4.6)$ & 5.1 & $(3.7-6.8)$ & 1.33 & 0.002 & $1.11-1.58$ & 1.26 & 0.030 & $1.02-1.56$ \\
\hline Serum creatinine & 77 & $(65-88.3)$ & 102 & $(82.3-130)$ & 1.03 & 0.001 & $1.01-1.04$ & 1.02 & 0.006 & $1.01-1.04$ \\
\hline Serum AST & 114 & $(70-195)$ & 156.5 & $(96.5-516)$ & 1.01 & 0.017 & $1.00-1.04$ & 1.01 & 0.019 & $1.00-1.04$ \\
\hline Serum ALT & 74 & $(38-121)$ & 92 & (55.8-293.3) & 1.01 & 0.012 & $1.001-1.06$ & 1.01 & 0.017 & $1.001-1.06$ \\
\hline Serum protein & 67 & $(63-72)$ & 66 & $(60.5-68.5)$ & 0.89 & 0.016 & $0.79-0.98$ & 0.88 & 0.022 & $0.79-0.98$ \\
\hline Serum albumin & 37 & $(35-40)$ & 37 & $(31-39)$ & 0.8 & 0.004 & $0.69-0.93$ & 0.81 & 0.006 & $0.70-0.94$ \\
\hline
\end{tabular}
compared to non-ICU (controls) patients

DPF- Days Post Fever; DPP- Days Post Presentation; LOS- Length of Stay; COR- Conditional Odds Ratio; IQR- Interquartile range.

*Adjusted Conditional Odds Ratio (ACOR) was obtained from a multivariate conditional logistic regression with test variables being adjusted by age and diabetes mellitus. Data in bold are risk factors involved in the most optimal predictive model of ICU requirement at first presentation. 
For each individual i, $x_{1}$ to $x_{3}$ are indicator variables taking on respective values, where $x_{1}$ represents neutrophil proportion (\%), $x_{2}$ represents ALT (IU/L) and $x_{3}$ represents serum urea $(\mathrm{mmol} / \mathrm{L})$.

Early ICU risk factors at $\mathbf{2 4}$ hours prior to ICU requirement Close monitoring of high risk patients in hospital for ICU requirement may be challenging as clinical conditions can evolve unpredictably. We further explored potential risk factors that can provide stratification at 24 hours prior to ICU requirement after their first presentation in hospital. Pulse rate $(\mathrm{COR}=1.07 ; 95 \% \mathrm{CI}=$ $1.002-1.14)$, white cell count $(\mathrm{COR}=1.59 ; 95 \% \mathrm{CI}=1.04$ $2.44)$, lymphocyte $(\mathrm{COR}=1.19 ; 95 \% \mathrm{CI}=1.03-1.38)$ and monocyte proportions $(\mathrm{COR}=1.35 ; 95 \% \mathrm{CI}=1.02-1.78)$ were observed to be significantly higher in ICU dengue patients 24 hour before ICU requirement compared with these at first presentation (Table 4). On the contrary, blood pressure $(\mathrm{COR}=0.96 ; 95 \% \mathrm{CI}=0.92-0.996)$ and hematocrit $(\mathrm{COR}=0.81 ; 95 \% \mathrm{CI}=0.66-0.99)$ were observed to be significantly lower in ICU dengue patients 24 hour prior to ICU requirement compared with that at the first presentation (Table 4). The most optimal model derived was a combination of the monocyte and lymphocyte proportion, blood pressure and pulse rate, which had an AUC of 0.94 (Hosmer-Lemeshow Goodness of fit test $P=0.96$ ), indicating that the model had good predictive potential of a high risk dengue-infected patient who is likely to require ICU in the next 24 hour. In addition, this model is more efficient than the model that is comprised of only pulse rate and blood pressure, which are known critical factors for ICU requirement, and has an AUC of only 0.74. A prognostic index $(P)$ was thus, created using the model with monocyte and lymphocyte proportion, blood pressure and pulse rate. Using the default cut-off of $P=0$, the model had sensitivity of $74.1 \%$, specificity of $88 \%$, PPV of $87 \%$ and NPV of $75.9 \%$. Using a cut-off of $P=-0.4$ (maximizing sensitivity and specificity), the model had sensitivity of $88.9 \%$, specificity of $76 \%$, PPV of $80 \%$ and NPV of $86.4 \%$. The predicted probability of ICU requirement in the next 24 hour $\left(p_{\mathrm{i}}\right)$ was calculated as follows:

$$
\begin{gathered}
P_{24 \text { HpriorICU }}=\ln \left(\frac{p i}{1-p i}\right)=0.333 x_{1 i}-0.105 x_{2 i}+0.079 x_{3 i} \\
+0.188 x_{4 i}-5.284
\end{gathered}
$$

For each individual i, $x_{1}$ to $x_{3}$ are indicator variables taking on respective values, where represents $x_{1}$ monocyte proportion (\%), $x_{2}$ blood pressure $(\mathrm{mHg}), x_{3}$ pulse rate $(\mathrm{bpm})$ and $x_{4}$ lymphocyte proportion (\%).

\section{Differential clinical and laboratory features of death cases} at first presentation among the ICU cases

A subgroup analysis was performed among the ICU cases to explore the significantly different clinical and laboratory features of death cases at first presentation in hospital (Table 5; Additional file 1: Table S1). The median age for death ICU cases $(n=7)$ and non-death ICU $(\mathrm{n}=20)$ subgroups was 48 (IQR: $27-50.5)$ and 43 (IQR: 36.8-54.3) years old, respectively. There were about $30 \%$ female in both the death and non-death subgroups $(P=1$; Additional file 1: Table S1). Based on the 2009 WHO classification, $100 \%$ and $85 \%$ had severe dengue in the death and non-death subgroups, respectively $(P=0.545$; Additional file 1: Table S1). The median length of stay in ICU was 3 and 3.5 days for the death and non-death subgroups, respectively $(P=0.383$; Additional file 1 : Table $\mathrm{S} 1)$. The median length of stay in hospital was 4 and 9 days for death and non-death subgroups $(P=0.029)$. Importantly, the following characteristics at first presentation were observed to be significantly different between the death and non-death ICU subgroups: having any warning signs $(P=$ $0.022)$, monocyte $(P=0.035)$ and eosinophils proportions $(P=0.035)$, serum AST $(P=0.049)$ and ALT $(P=0.010)$, and protein $(P=0.039)$ (Table 5$)$.

\section{Discussion}

Dengue infection results in a wide spectrum of clinical severity, from self-limiting dengue fever to severe dengue. Timely appropriate monitoring and clinical management,

Table 4 Significantly associated laboratory variables at $\mathbf{2 4} \mathrm{h}$ prior to ICU requirement compared to first presentation

\begin{tabular}{|c|c|c|c|c|c|c|c|}
\hline Variables & $\begin{array}{l}\text { Den-infected ICU at } \\
\text { presentation }(n=23)^{\wedge}\end{array}$ & (IQR) & $\begin{array}{l}\text { Den-infected ICU at } 24 \mathrm{H} \\
\text { prior ICU }(n=23)^{\wedge}\end{array}$ & (IQR) & COR & P-value & $95 \% \mathrm{Cl}$ \\
\hline Systolic blood pressure & 110 & $(91.5-118.5)$ & 90 & $(80-101.5)$ & 0.96 & 0.03 & $0.92-0.996$ \\
\hline Pulse rate & 95.5 & $(88.5-102)$ & 101 & $(90.5-117.5)$ & 1.07 & 0.044 & $1.002-1.14$ \\
\hline White cell count & 3.3 & $(2.4-4.2)$ & 4.9 & $(3-7.4)$ & 1.59 & 0.033 & $1.04-2.44$ \\
\hline Proportion lymphocytes & 16.5 & $(9.8-19.9)$ & 23.5 & $(18.1-28.6)$ & 1.19 & 0.020 & $1.03-1.38$ \\
\hline Proportion monocytes & 7.9 & $(5.1-9.9)$ & 12.1 & $(9.1-17.9)$ & 1.35 & 0.033 & $1.02-1.78$ \\
\hline Serum hematocrit & 44.9 & $(42.8-47.2)$ & 40.2 & (33.6-43.6) & 0.81 & 0.037 & $0.66-0.99$ \\
\hline
\end{tabular}
of dengue-infected ICU (cases) patients

COR- Conditional Odds Ratio; IQR- Interquartile range.

$\wedge$ - Four cases were excluded as they were admitted into ICU less than 24 hours after first presentation at hospital. Data in bold are risk factors involved in the most optimal predictive model of ICU requirement at $24 \mathrm{~h}$ prior to ICU requirement. 
Table 5 Significantly different characteristics between deaths and non-deaths at first presentation in the subgroup analysis of ICU dengue-infected patients

\begin{tabular}{|c|c|c|c|c|c|}
\hline Variables & $\begin{array}{l}\text { Den-infected ICU } \\
\text { non-death }(n=20)\end{array}$ & $\% /(I Q R)$ & $\begin{array}{l}\text { Den-infected ICU } \\
\text { death }(n=7)\end{array}$ & $\% /(I Q R)$ & P-value* \\
\hline Median LOS in hospital & 9 & $(7-17.25)$ & 4 & $(3.5-8.5)$ & 0.0285 \\
\hline Median DPF to death & 0 & & 3 & $(2.5-7.5)$ & \\
\hline Median DPP to death & 0 & & 7 & $(7-15.5)$ & \\
\hline \multicolumn{6}{|l|}{ Any warning sign } \\
\hline Yes & 9 & 45 & 7 & 100 & $0.022 \wedge$ \\
\hline Proportion monocytes & 9.1 & $(6.55-12.55)$ & 5.4 & $(3.8-7.15)$ & 0.0348 \\
\hline Proportion eosinophils & 0.1 & $(0-0.4)$ & 0.5 & $(0.4-0.9)$ & 0.0351 \\
\hline Serum AST & 11 & $(10-17)$ & 19 & $(16-29)$ & 0.0487 \\
\hline Serum ALT & 126 & $(70.25-263.25)$ & 939 & $(442.75-1577.75)$ & 0.0103 \\
\hline Serum protein & 85.5 & $(39.25-146.25)$ & 491 & $(159-653.5)$ & 0.0328 \\
\hline
\end{tabular}

$\wedge$ - Fisher's exact test.

*-Wilcoxon rank-sum test.

IQR- Interquartile range.

mainly with fluid interventions of dengue patients is critical to reduce morbidity and mortality $[2,3]$. Some of these patients may also require critical clinical management in intensive care unit (ICU). Healthcare resources such as ICU beds and close monitoring may be limited during a large epidemic, making effective triage of patients who may require ICU requirement more crucial. However, there is currently a lack of systematic study looking at potential early risk factors for ICU requirement. Early clinical and laboratory risk factors during first presentation at hospital or even 24 hours prior to ICU requirement via regular strict monitoring of risk factors, may assist clinicians to triage these high-risk patients.

From this matched case-control study, dengue patients who were either between 50 to 59 years old or with pre-existing diabetes had about five times the risk of ICU requirement, compared with dengue patients who are less than 30 years old or without diabetes, respectively (Table 1). Age group 50 to 59 years old was also implicated in ICU requirement in a case-control study of dengue fatality [17] and the recent multi-center mortality cohort study in Singapore [12]. However, the age risk factor of dengue hemorrhagic fever (DHF) observed in a dengue 2 epidemic is age group of 30-49 years old [18]. This suggests that different severity, either defined as DHF, fatality or the requirement for ICU requirement, may affect different age groups. Furthermore, age group of 60 years old and above was not observed to be a risk factor of ICU requirement previously [19,20], which supported the observation in this study. On the contrary, it was observed that the mean age of ICU requirement in New Delhi case series was younger (39.6 years) [4], with about only $10 \%$ of the ICU dengue patients aged greater than 55 years, compared with the $22 \%$ in this study. In addition, in southern Taiwan, dengue patients
65 years old or above were reported to have a higher fatality risk compared with patients less than 65 years of age [20]. Interestingly, there was no significant difference in age between severe cases requiring ICU and benign cases in a dengue serotype 1 epidemic in New-Caledonia (South Pacific), with mean age of 47 years in both groups [5]. These findings highlighted the potential differences in epidemiology of ICU dengue patients in different geographical regions, which would be interesting for further evaluation. In general, the risk of ICU requirement was observed to increase from age 40 years old and above (Table 1). Diabetes was not an independent risk factor of ICU requirement in this study (even after adjusting for DHF; data not shown), which is also similarly reported in dengue mortality studies $[12,17,21]$. However, diabetes was shown to be an independent risk factor of DHF as an outcome $[18,22]$, and DHF as an outcome was significantly associated with ICU requirement in this study. This may suggest that having diabetes may not predispose a patient to ICU requirement or death directly, but it may increase the risk of DHF, potentially resulting in ICU requirement. However, caution in interpretation remains as the sample size of cases was small in this study. Furthermore, IgG status was not significantly associated with ICU requirement, hence, secondary infection is not likely to confound the associations observed in this study.

Based on the WHO classification at presentation, patients who fulfilled the WHO 2009 severe dengue classification were at a higher risk of ICU requirement, eight times more than patients classified as non-severe, but not for the WHO 1997 DHF/DSS criteria at first presentation (Table 2). This suggests that the WHO 2009 classification for severe dengue is more sensitive in identifying clinically more severe patients than that of the WHO 1997 classification for DHF/DSS at first presentation, which is 
also reported in other studies [10,12,23]. However, both the WHO 1997 and 2009 classification systems only achieved sensitivity of about $19 \%$ and $52 \%$, respectively, in differentiating patients who required ICU requirement at first presentation. On the other hand, both classification systems achieved high specificity of $87 \%$ and $94 \%$, respectively. In contrast, as final outcome, the WHO 1997 classification system achieved sensitivity and specificity of about $67 \%$ and $78 \%$ respectively, and the WHO 2009 classification system achieved sensitivity and specificity of about $89 \%$ and $90 \%$ respectively. This is comparable to the sensitivity and specificity of identifying patients requiring category III care (which includes ICU requirement) for DHF/ DSS (39.0\% and $75.5 \%$, respectively) and for severe dengue ( $92.1 \%$ and $78.5 \%$, respectively) as described by Narvaez F et al. [10].

Most warning signs and symptoms at first presentation in this study were not observed as risk factors of ICU requirement in this study, except for hypoproteinemia, hypotension, and hematocrit change $\geq 20 \%$ concurrent with platelet $<50 \mathrm{~K}$ (Table 3 ). Other studies also reported that most warning signs were not able to identify patients progressing into DHF, severe dengue, category III care or fatality efficiently $[9,10,20]$. However, gastrointestinal symptoms were proposed as an important warning sign for severe dengue and fatality [20,22], but it was not associated with ICU requirement in this study. Hypoproteinemia was observed in most $(>80 \%)$ of the fatal cases in other studies [12,24], and was observed at first presentation of severe patients who required ICU requirement [5]. Similarly, hypotension was observed in $71 \%$ of fatal cases [17], and was observed to be significantly more in severe cases who required ICU [5]. Interestingly, having any warning signs at first presentation is also a risk factor for death among ICU cases (Table 5), besides for severe dengue [25].

The following laboratory parameters at presentation may be potential indicators of ICU requirement at first presentation in hospital, and were reported in ICU requirement case series and/or fatality case-control studies: pulse rate [17], neutrophil [5], lymphocytes and monocytes proportions, platelet count $[6,7,17,21,24]$, serum urea, creatinine $[5,7,17]$, AST, ALT $[5-7,17,24]$, protein $[5,7]$ and albumin $[5,7,17,24]$. In our study, a prognostic model using a combination of neutrophil proportion, ALT and serum urea level provided a sensitivity of $88.2 \%$, specificity of $88.9 \%$, PPV of $62.5 \%$ and NPV of $97.3 \%$. This is comparable to the models for predicting severity, defined as death using a model comprised of creatinine, free bilirubin, amylase and platelets with PPV 66.7\% and NPV $75.5 \%$ [5], and the prediction of DHF in adult patients at presentation with median DPF of 5 days using a model comprised of clinical bleeding, serum urea, serum protein, and lymphocyte proportion with sensitivity $83 \%$, specificity
84\%, PPV 18\%, NPV 99\% [26-28]. Serum urea level was a common variable in these models, which suggests urea level in serum may be an important risk factor for severity. However, the level of serum urea is influenced by bleeding, renal failure, and changes in hemoconcentration. Hence, further research is still required to elucidate the impact of urea on dengue severity, which was shown previously to increase complement-fixation of dengue antigen [29]. Monocyte proportion, AST, ALT and serum proteins were also observed to be significantly different between the death and non-death among ICU cases. This suggests that these could be important factors involved in the progression of fatality after ICU requirement, and should be considered for close monitoring during hospitalization. On the contrary, only bandemia was highlighted as a significant risk factor at first presentation between non-fatal and fatal patients [21].

Pulse rate, lymphocyte and monocyte proportions, blood pressure, white cell count, and hematocrit were observed as early risk factors at 24 hours prior to ICU requirement, and were reported in ICU requirement case series and/or fatality case-control studies $[4,17,21]$. In the fatality study, leukocytosis and platelet count were also shown to be significantly different between the first presentation and pre-fatal data [21]. Interestingly, lymphocyte and monocyte proportions of ICU dengue patients at 24 hours prior to ICU requirement was significantly greater compared with that at first presentation, but lower compared with non-ICU dengue patients at first presentation. This may suggest the dynamic differences in immune response between dengue patients who required ICU requirement and dengue patients who did not require ICU requirement were already evident even at first presentation, which is about three to five days post fever. In addition, this may reflect the observation of the immune response changes rapidly over time as a patient progresses from first presentation at hospital to 24 hours before ICU requirement in another study [30]. However, further research is required to validate these observation. A prognostic model using a combination of monocytes and lymphocytes proportion, blood pressure and pulse rate provided a sensitivity of $74.1 \%$, specificity of $88 \%$, PPV of $87 \%$ and NPV of $75.9 \%$. Some of these significant variables were also part of the model used for predicting pediatric DHF which comprised of white cell count, monocytes proportion, platelet count, and hematocrit with sensitivity of $97 \%$ and specificity of $48 \%$ [31]. Furthermore, this model is clinically relevant as pulse rate and blood pressure were known critical factors for ICU requirement, and were selected unbiasedly based on its significance by the regression techniques. Furthermore, this model was observed to be more efficient as compared to the predictive model with only pulse rate and blood pressure as significant variables. 
There are some limitations in our study that should be taken into consideration when interpreting the results. First, the study had a relatively small number of patients with ICU requirement that may result in low statistical power to detect true association. However, the power was maximized by using four matched controls to each case. Furthermore, it should be noted that Tan Tock Seng Hospital is a major infectious disease center in Singapore, which provided care for about $40 \%$ of all reported dengue patients in Singapore. Second, no dengue serotype data were available for individual study subjects. In order to account for the serotype variable, we used the year of dengue presentation as a surrogate for serotype (as describe in Methods) as well as a matching factor for our selection of controls to minimize confounding effect due to the different predominant serotype each year. Third, there were no data on pharmaceutical intervention prior and during hospitalization to account for potential contribution to disease severity due to chronic diseases. However, since both the ICU and non-ICU dengue patients did not have significant differences in preexisting comorbidities, the bias due to pharmaceutical interventions on severity outcome should be minimal. Fourth, this model is derived from adult dengue patients presenting to hospital at a median of $5 \mathrm{DPF}(\mathrm{IQR}=3-5$ days). Hence, the model may be less accurate when used for triage of patients outside this period range, or used at the community healthcare facilities, which may have much shorter days post fever. Further validation studies are still required to confirm the validity and reliability of this model. Fifth, three parameters in our probability equation were laboratory test results, which may not be easily available in resource-poor countries. However, it is hopeful that as the awareness of dengue prevention and clinical management is raised with the guidance of the WHO, there will be an increased in public-private partnerships that can help to resolve this challenge gradually. Sixth, the lack of APACHE II or SOFA scores may have further limit the accuracy and performance of these model development. Lastly, these risk factors and models would not be as useful as expected if there is a lack of commitment of all hospital physicians, particularly in emergency units, to ensure close monitoring of patients during hospitalization using the risk factors identified in this study.

\section{Conclusion}

Studies to assess early risk factors for severity as defined by DHF/DSS and severe dengue for effective triage are important. However, dengue severity as defined by the requirement of ICU is also critical, but lacking. This is the first systematic study, to our best knowledge, that highlighted the differential pathophysiological background of dengue patients at presentation and at 24 hours prior to ICU requirement. Demographic and clinical risk factors for triage at first presentation comprised of age group 50 to 59 years old, pre-existing diabetes, the WHO 2009 classification at first presentation, hypoproteinemia, hypotension and hematocrit change $\geq 20 \%$ concurrent with platelet $<50 \mathrm{~K}$ were proposed. For laboratory risk factors, two optimal models with significantly associated variables were derived and proposed to complement the WHO classification system. An optimal model at first presentation is comprised of neutrophil proportion, ALT and serum urea level, and another optimal model for assessing severity 24 hours prior to ICU requirement is comprised of monocytes and lymphocytes proportions, blood pressure and pulse rate. This would be useful to identify dengue patients who may require ICU requirement early at presentation as well as during hospitalization for closer monitoring and clinical management to reduce morbidity and mortality, when validated in independent larger studies.

\section{Additional files}

Additional file 1: Table S1. Subgroup analysis of characteristics of death and non-death cases among ICU patients at first presentation. This table shows the overall characteristics at first presentation of death cases compared to non-death cases among ICU patients that were insignificantly different.

Additional file 2: Table S2. Signs and symptoms at first presentation of dengue-infected non-ICU (controls) and ICU (cases) patients. This table shows the signs and symptoms that were insignificantly associated to ICU at first presentation in hospital.

\section{Abbreviations}

ICU: Intensive care unit; Cl: Confidence interval; WHO: World Health Organization; DF: Dengue fever; DHF: Dengue hemorrhagic fever; DSS: Dengue shock syndrome; TTSH: Tan Tock Seng Hospital; COR: Conditional odds ratio; ACOR: Adjusted conditional odds ratio; ARDS: Acute respiratory disease syndrome; PCR: Polymerase chain reactions; Pctl: Percentiles; AST: Aspartate aminotransferases; ALT: Alanine aminotransferases; PPV: Positive predictive value; NPV: Negative predictive value.

\section{Competing interests}

The authors declare that they have no competing interests.

\section{Authors' contributions}

PJ, YSL, DCL conceived the study, and participated in the design of the study. TLT, YSL, DCL were involved in data collection and coordination. PJ performed the statistical analysis and drafted the manuscript. PJ, TLT, YSL, DCL helped to review the manuscript. All authors read and approved the final manuscript.

\section{Acknowledgement}

We are grateful to Ms. Adriana S Tan for data entry, extraction and management.

\section{Funding}

This work was supported by the National Medical Research Council grant NMRC/TCR/005/2008. The funders had no role in study design, data collection and analysis, decision to publish, or preparation of the manuscript.

\section{Author details}

${ }^{1}$ Saw Swee Hock School of Public Health, National University of Singapore, Singapore, Singapore. ${ }^{2}$ Infectious Diseases, Genome Institute of Singapore, Singapore, Singapore. ${ }^{3}$ Communicable Disease Center, Institute of Infectious Diseases and Epidemiology, Tan Tock Seng Hospital, Singapore, Singapore. ${ }^{4}$ Department of Medicine, Yong Loo Lin School of Medicine, National University of Singapore, Singapore, Singapore. ${ }^{5}$ Lee Kong Chian School of Medicine, Nanyang Technological University, Singapore, Singapore. 
Received: 31 December 2013 Accepted: 21 November 2014 Published online: 05 December 2014

\section{References}

1. World Health Organization: Global Strategy for Dengue Prevention and Control, 2012-2020. Geneva: World Health Organization; 2012

2. World Health Organization: Dengue Haemorrhagic Fever: Diagnosis, Treatment, Prevention and Control. 2nd edition. Geneva: World Health Organization; 1997

3. World Health Organization: Dengue: Guidelines for Diagnosis, Treatment, Prevention and Control. Geneva: World Health Organization; 2009.

4. Juneja D, Nasa P, Singh O, Javeri Y, Uniyal B, Dang R: Clinical profile, intensive care unit course, and outcome of patients admitted in intensive care unit with dengue. J Crit Care 2011, 26(5):449-452.

5. Bouldouyre MA, Baumann F, Berlioz-Arthaud A, Chungue E, Lacassin F: Factors of severity at admission during an epidemic of dengue 1 in New Caledonia (South Pacific) in 2003. Scand J Infect Dis 2006, 38(8):675-681.

6. Chandralekha, Gupta P, Trikha A: The North Indian dengue outbreak 2006: a retrospective analysis of intensive care unit admissions in a tertiary care hospital. Trans R Soc Trop Med Hyg 2008, 102(2):143-147.

7. Schmitz L, Prayag S, Varghese S, Jog S, Bhargav-Patil P, Yadav A, Salunke D, Vincent $J$ : Nonhematological organ dysfunction and positive fluid balance are important determinants of outcome in adults with severe dengue infection: a multicenter study from India. J Crit Care 2011 26(5):441-448.

8. Lee LK, Earnest A, Carrasco LR, Thein TL, Gan VC, Lee VJ, Lye DC, Leo YS: Safety and cost savings of reducing adult dengue hospitalization in a tertiary care hospital in Singapore. Trans R Soc Trop Med Hyg 2013, 107(1):37-42.

9. Thein TL, Gan VC, Lye DC, Yung CF, Leo YS: Utilities and limitations of the World Health Organization 2009 warning signs for adult dengue severity. PLoS Negl Trop Dis 2013, 7(1):e2023.

10. Narvaez F, Gutierrez G, Perez MA, Elizondo D, Nunez A, Balmaseda A, Harris E: Evaluation of the traditional and revised WHO classifications of Dengue disease severity. PLOS Negl Trop Dis 2011, 5(11):e1397.

11. Lee KS, Lai YL, Lo S, Barkham T, Aw P, Ooi PL, Tai JC, Hibberd M, Johansson P, Khoo SP, Ng LC: Dengue virus surveillance for early warning, Singapore. Emerg Infect Dis 2010, 16(5):847-849.

12. Leo YS, Thein TL, Fisher DA, Low JG, Oh HM, Narayanan RL, Gan VC, Lee VJ, Lye DC: Confirmed adult dengue deaths in Singapore: 5-year multicenter retrospective study. BMC Infect Dis 2011, 11:123.

13. Sedgwick P: Why match in case-control studies? BMJ 2012, 344:e691.

14. Sedgwick P: Confounding in case-control studies. BMJ 2010, 341:c5136.

15. Sedgwick P: Analysing case-control studies: adjusting for confounding. BMJ 2013, 346:f25

16. Ministry of Health $\mathrm{S}$ : Review of dengue serotype surveillance programme in Singapore. Epidemiol News Bull 2012, 38(3):54-61.

17. Ong A, Sandar M, Chen MI, Sin LY: Fatal dengue hemorrhagic fever in adults during a dengue epidemic in Singapore. Int J Infect Dis 2007, 11(3):263-267.

18. Pang J, Salim A, Lee VJ, Hibberd ML, Chia KS, Leo YS, Lye DC: Diabetes with hypertension as risk factors for adult dengue hemorrhagic fever in a predominantly dengue serotype 2 epidemic: a case control study. PLOS Negl Trop Dis 2012, 6(5):e1641.

19. Lye DC, Lee VJ, Sun Y, Leo YS: The benign nature of acute dengue infection in hospitalized older adults in Singapore. Int J Infect Dis 2010, 14(5):e410-e413.

20. Lee IK, Liu JW, Yang KD: Clinical and laboratory characteristics and risk factors for fatality in elderly patients with dengue hemorrhagic fever. AmJTrop Med Hyg 2008, 79(2):149-153.

21. Lee IK, Liu JW, Yang KD: Fatal dengue hemorrhagic fever in adults: emphasizing the evolutionary pre-fatal clinical and laboratory manifestations. PLoS Negl Trop Dis 2012, 6(2):e1532

22. Figueiredo MA, Rodrigues LC, Barreto ML, Lima JW, Costa MC, Morato V, Blanton R, Vasconcelos PF, Nunes MR, Teixeira MG: Allergies and diabetes as risk factors for dengue hemorrhagic fever: results of a case control study. PLOS Negl Trop Dis 2010, 4(6):e699.

23. Gan VC, Lye DC, Thein TL, Dimatatac F, Tan AS, Leo YS: Implications of discordance in world health organization 1997 and 2009 dengue classifications in adult dengue. PLoS One 2013, 8(4):e60946.
24. Sam SS, Omar SF, Teoh BT, Abd-Jamil J, AbuBakar S: Review of dengue hemorrhagic fever fatal cases seen among adults: a retrospective study. PLoS Negl Trop Dis 2013, 7(5):e2194.

25. Leo YS, Gan VC, Ng EL, Hao Y, Ng LC, Pok KY, Dimatatac F, Go CJ, Lye DC: Utility of warning signs in guiding admission and predicting severe disease in adult dengue. BMC Infect Dis 2013, 13:498.

26. Lee VJ, Lye DC, Sun Y, Fernandez G, Ong A, Leo YS: Predictive value of simple clinical and laboratory variables for dengue hemorrhagic fever in adults. J Clin Virol 2008, 42(1):34-39.

27. Lee VJ, Lye DC, Sun Y, Leo YS: Decision tree algorithm in deciding hospitalization for adult patients with dengue haemorrhagic fever in Singapore. Trop Med Int Health 2009, 14(9):1154-1159.

28. Thein TL, Leo YS, Lee VJ, Sun Y, Lye DC: Validation of probability equation and decision tree in predicting subsequent dengue hemorrhagic fever in adult dengue inpatients in Singapore. Am J Trop Med Hyg 2011, 85(5):942-945.

29. Cornesky RA, Hammon WM, Atchison RW, Sather GE: Effect of urea on the hemagglutinating and complement-fixing antigens of type 2 Dengue virus. Infect Immun 1972, 6(6):952-957.

30. Potts JA, Gibbons RV, Rothman AL, Srikiatkhachorn A, Thomas SJ, Supradish PO, Lemon SC, Libraty DH, Green S, Kalayanarooj S: Prediction of dengue disease severity among pediatric Thai patients using early clinical laboratory indicators. PLoS Negl Trop Dis 2010, 4(8):e769.

31. Hoang LT, Lynn DJ, Henn M, Birren BW, Lennon NJ, Le PT, Duong KT, Nguyen TT, Mai LN, Farrar JJ, Hibberd ML, Simmons CP: The early wholeblood transcriptional signature of dengue virus and features associated with progression to dengue shock syndrome in Vietnamese children and young adults. J Virol 2010, 84(24):12982-12994.

doi:10.1186/s12879-014-0649-2

Cite this article as: Pang et al:: Early clinical and laboratory risk factors of intensive care unit requirement during 2004-2008 dengue epidemics in Singapore: a matched case-control study. BMC Infectious Diseases 2014 14:649.

\section{Submit your next manuscript to BioMed Central and take full advantage of:}

- Convenient online submission

- Thorough peer review

- No space constraints or color figure charges

- Immediate publication on acceptance

- Inclusion in PubMed, CAS, Scopus and Google Scholar

- Research which is freely available for redistribution 